\title{
Atributos químicos e físicos do solo cultivado com forrageiras invernais e soja em sucessão
}

\section{Chemical and physical soil attributes cultivated with winter forage and soybean in succession}

Andressa Perini Vengen

Universidade Estadual do Oeste do Paraná

E-mail: andressaperini@hotmail.com

OrcID: https://orcid.org/0000-0001-5143-9329

Eloisa Mattei

Universidade Estadual do Oeste do Paraná

E-mail: eloisa-mattei@hotmail.com

OrclD: https://orcid.org/0000-0002-0010-5385

Carlos Augusto Rocha de Moraes Rego

Universidade Estadual do Oeste do Paraná

E-mail: cassielcarlos@hotmail.com

OrcID: https://orcid.org/0000-0003-4414-1449

Jonas Francisco Egewarth

Universidade Estadual do Oeste do Paraná

E-mail: jonas.egewarth@gmail.com

OrcID: https://orcid.org/0000-0002-8456-9194

Marinez Carpiski Sampaio

Universidade Estadual do Oeste do Paraná

E-mail: mari_marinez@hotmail.com

OrcID: https://orcid.org/0000-0001-7712-2127

Paulo Sérgio Rabello de Oliveira Universidade Estadual do Oeste do Paraná

Campus Marechal Cândido Rondon

E-mail: rabello.oliveira@hotmail.com

OrclD: https://orcid.org/0000-0002-0478-1006

Resumo: Muitos produtores resistem em adotar a presença dos animais nas lavouras, pois acreditam que possa compactar o solo e causar redução na produtividade da cultura em sucessão. Diante disso, objetivou-se avaliar os atributos químicos e físicos do solo após o cultivo de forrageiras invernais sob manejos de pastejo e soja em sucessão em sistema de integração lavoura-pecuária. O experimento foi conduzido em um LATOSSOLO VERMELHO sob o delineamento de blocos ao acaso, em esquema de faixas, com três repetições. Foram testados diferentes forrageiras invernais (aveia BRS 139, aveia preta IPR 61, aveia branca IPR Esmeralda, Triticale 111 Tpolo e o consórcio de Triticale 111 TPolo e aveia IPR Esmeralda), manejos de pastejo (sem pastejo, um pastejo e dois pastejos) e profundidades do solo (0-0,05, 0,05-0,10 e 0,10-0,20 m). Foram determinados os seguintes atributos: nitrogênio total (NT), carbono orgânico total (COT), relação carbono e nitrogênio $(\mathrm{C} / \mathrm{N})$, matéria orgânica do solo $(\mathrm{MOS})$, porosidade total $(\mathrm{Pt})$, macroporosidade (Mac), microporosidade (Mic) e densidade aparente (Ds). As forrageiras utilizadas para pastejo no período de inverno resultaram em diferentes teores de MOS, NT e Mac. O cultivo do Triticale 111 TPolo resultou em menor Mic e Pt quando manejado sob dois pastejos nas camadas de 0,05-0,10 e 0,10-0,20 m e a maior Ds foi encontrada 
na camada superficial, independentemente da forrageira e manejo. As forrageiras invernais quando submetidas a pressões de pastejo não modificaram os atributos do solo entre as profundidades avaliadas.

Palavras-chave: Manejo do solo; Matéria orgânica do solo; Frequência de pastejo; Sistema de integração lavoura-pecuária.

Abstract: Many producers resist adopting the presence of animals on farms, as they believe that it can compact the soil and cause reduction in crop productivity in succession. Thus, it was aimed to assess the chemical and physical soil properties after cultivation of winter forage under different managements grazing and soybean crop in integrated crop-livestock system. The experiment was carried out in a Red Latosol on a randomized block design, in strip plots and three replications. Were tested different winter forages (oat BRS 139, black oat IPR 61, white oat IPR Esmeralda, Triticale 111 Tpolo and the consortium of Triticale 111 TPolo and oat IPR Emerald), grazing managements (without grazing, one grazing and two grazing) and soil layers $(0-0.05,0.05-0.10$, and 0.10-0.20 m). Total nitrogen (NT), total organic carbon (TOC), carbon and nitrogen ratio (C/N), soil organic matter (MOS), total porosity (Pt), macroporosity (Mac), microporosity (Mic) and apparent density (Ds) were determined. The forage plants used for grazing in the winter period resulted in different levels of MOS, NT and Mac. The cultivation of Triticale 111 TPolo resulted in lower Mic and Pt when managed under two grazing in the $0.05-0.10$ and $0.10-0.20 \mathrm{~m}$ layers and the highest Ds was found in the surface layer, regardless of the forage and management. The winter forages when submitted to different grazing pressures did not change the soil attributes between the evaluated layers.

Keywords: Soil management; Organic soil matter; Grazing frequency; Integrated crop-livestock system.

Data de recebimento: 02/05/2020

Data de aprovação: 09/06/2020

DOI: https://doi.org/10.30612/agrarian.v13i50.11832

\section{Introdução}

A adoção de sistemas produtivos que integram a agricultura e a pecuária numa mesma área, comumente chamado de sistema integrado de produção agropecuária (SIPA), vem se tornando comum em diversas regiões do Brasil (CARVALHO et al., 2016; GLÉRIA et al., 2017; REGO et al., 2018; RODRIGUES et al., 2019). Segundo Balbino et al. (2019), os SIPA podem ser classificados e definidos de diferentes maneiras, sendo a adoção da integração lavoura-pecuária (Agropastoril) em plantio direto a mais amplamente empregada dentro do Brasil (CARVALHO et al., 2014).

A integração lavoura-pecuária (ILP) se destaca como uma opção viável para a produção, pois além de ser uma prática conservacionista que auxilia na recuperação do solo e no controle de pragas e doenças ainda pode diversificar a produção na propriedade diminuindo riscos e aumentando os lucros (KUNRATH et al., 2015; REGO et al., 2018; MORAES REGO et al., 2020). O tipo de manejo, textura do solo, teor de umidade, teor de matéria orgânica, espécie vegetal, clima, entre outros, refletem diretamente na qualidade química, física e biológica do solo (COSTA et al., 2015).

Apesar das inúmeras vantagens da ILP, para seu sucesso é necessário o conhecimento aprofundado do sistema como um todo, ou seja, a interação solo-planta-animal, sendo esses componentes dinâmicos e que interagem entre si (GLÉRIA et al., 2017). Nesse contexto, o uso de indicadores que retratam processos que ocorrem na interface solo-raiz é determinante para avaliar o impacto do manejo na qualidade do solo (MOREIRA et al., 2014), como a matéria orgânica, o carbono orgânico total, o nitrogênio total do solo, a porosidade do solo e a densidade.

As forrageiras invernais são importantes fontes de cobertura do solo e alternativa de alimentação animal no período de inverno, trazendo consigo diversos benéficos (PIANO et al., 2015; GLÉRIA et al., 2017). Entretanto, muitos produtores resistem em adotar a entrada de animais na área, pois acreditam que isso possa compactar o solo e causar redução na produtividade das culturas em sucessão. Essa preocupação é relevante, pois o pastejo intensivo podem reduzir o aporte de matéria orgânica do solo (MOREIRA et al., 2014; BORTOLINI

Agrarian, Dourados, v. 13, n. 50, p. 467- 477, 2020. 
et al., 2016), ter efeito adverso sobre a física do solo e alteração sobre o sistema poroso (KUNZ et al., 2013). Vários estudos têm demonstrado essa interferência negativa na qualidade do solo (MOREIRA et al., 2014; ORTIGARA et al., 2014).

Vale ressaltar que, a interferência do pisoteio animal nos atributos do solo é dependente de aspectos ligados a condições climáticas, a espécie animal, as condições edáficas, a carga animal utilizada e ao tempo de uso do sistema (BORTOLINI et al., 2016). Resultados que comprovam a ausência de prejuízos a qualidade do solo foram observados por Bortolini et al. (2016) ao estudarem os atributos físicos, dinâmica da água e teores de matéria orgânica de um Cambissolo húmico submetido a intensidades de pastejo de gado leiteiro.

Diante do exposto, o presente trabalho tem como hipótese que: o emprego de diferentes forrageiras invernais e o manejo de pastejo com sucessão de soja melhoram os atributos químicos e físicos de solo. Nesse contexto, o objetivo foi avaliar os atributos químicos e físicos do solo após o cultivo de forrageiras invernais sob manejos de pastejo e soja em sucessão em sistema de integração lavoura-pecuária.

\section{Material e Métodos}

O experimento foi conduzido na Fazenda Experimental "Professor Antônio Carlos dos Santos Pessoa", pertencente à Universidade Estadual do Oeste do Paraná - UNIOESTE, Campus Marechal Cândido Rondon, localizado na região Oeste do Paraná, latitude $24^{\circ} 31^{\prime} 58^{\prime \prime S}$ e longitude $54^{\circ} 01^{\prime} 10^{\prime \prime} \mathrm{W}$, com altitude aproximada de $400 \mathrm{~m}$.

O solo foi classificado como LATOSSOLO VERMELHO Eutroférrico (LVef) de textura muito argilosa (Tabela 1) (Santos et al., 2018) e segundo a classificação de Köppen o clima da região é do tipo Cfa, mesotérmico úmido subtropical de inverno seco, com chuvas bem distribuídas durante todo o ano e verões quentes com temperatura média anual entre 22 a $23^{\circ} \mathrm{C}$ e precipitação média anual de 1600 a 1800 milímetros (Alvares et al., 2014). Os dados meteorológicos referentes ao período experimental (Figura 1) foram obtidos junto da Estação Climatológica Automática do Núcleo de Estações Experimentais da UNIOESTE, localizada próximo à área experimental. As características químicas e físicas antes da implantação do experimento estão descritas na Tabela 1, estas realizadas conforme descrito na metodologia de Pavan et al. (1992).

Tabela 1. Caraterização química e física do solo, na camada de 0-10 e 10-20 cm de profundidade, antes da implantação das culturas de inverno.

\begin{tabular}{|c|c|c|c|c|c|c|c|c|c|c|c|c|c|}
\hline Cam & $\mathbf{P}$ & $\mathrm{pH}$ & $\mathrm{H}+\mathrm{Al}$ & $\mathrm{Al}^{3+}$ & $\mathbf{K}^{+}$ & $\mathrm{Ca}^{2+}$ & $\mathbf{M g}^{2+}$ & SB & СТC & $\mathbf{v}$ & Argila & Silte & Areia \\
\hline $\mathrm{cm}$ & $\begin{array}{c}\mathrm{mg} \\
\mathrm{dm}^{-3}\end{array}$ & $\mathrm{CaCl}_{2}$ & & \multicolumn{5}{|c|}{ - } & & $\%$ & \multicolumn{3}{|c|}{ 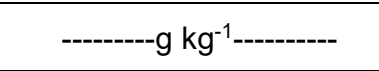 } \\
\hline $0-10$ & 22,8 & 4,7 & 6,6 & 0 & 0,3 & 3,3 & 1,4 & 4,9 & 9,5 & 51,6 & 681 & 266 & 52 \\
\hline $10-20$ & 30,3 & 4,6 & 6,8 & 0,3 & 0,1 & 3,3 & 1,4 & 4,9 & 10,3 & 47,4 & 751 & 199 & 49 \\
\hline
\end{tabular}

Cam: Camada. P e K - Extrator MEHLICH ${ }^{-1} ; \mathrm{Al}$, Ca e Mg $=\mathrm{KCl} 1 \mathrm{~mol} \mathrm{~L}^{-1} ; \mathrm{H}+\mathrm{Al}=\mathrm{pH} \operatorname{SMP}(7,5)$.

A área experimental já vinha sendo manejada há 5 anos sob SILP, com plantio direto consolidado. Durante os últimos 2 anos foram cultivados soja no verão e espécies de forrageiras solteiras ou consorciadas para pastejo de gado de leite no inverno. Anteriormente a implantação do experimento, realizou-se a coleta de solo a fim de identificar a necessidade de calagem e/ou adubação. As características químicas e físicas são descritas na Tabela 1.

O delineamento experimental foi de blocos casualizados, em esquema de faixas, com três repetições. As parcelas foram formadas pela combinação das faixas $A$ ( 5 níveis) e $B$ ( 3 níveis) com dimensões de $4 \times 13$ $\mathrm{m}\left(52 \mathrm{~m}^{2}\right)$, totalizando 45 parcelas experimentais e área total de $2340 \mathrm{~m}^{2}(0,234$ hectares $)$. As faixas $A$ compreenderam as forrageiras invernais: aveia preta BRS 139, aveia preta IPR 61, aveia branca IPR Esmeralda, Triticale 111 TPolo e o consórcio entre Triticale 111 TPolo e aveia branca IPR Esmeralda, e nas faixas $B$ foram alocados os manejos: sem pastejo, um pastejo e dois pastejos. 


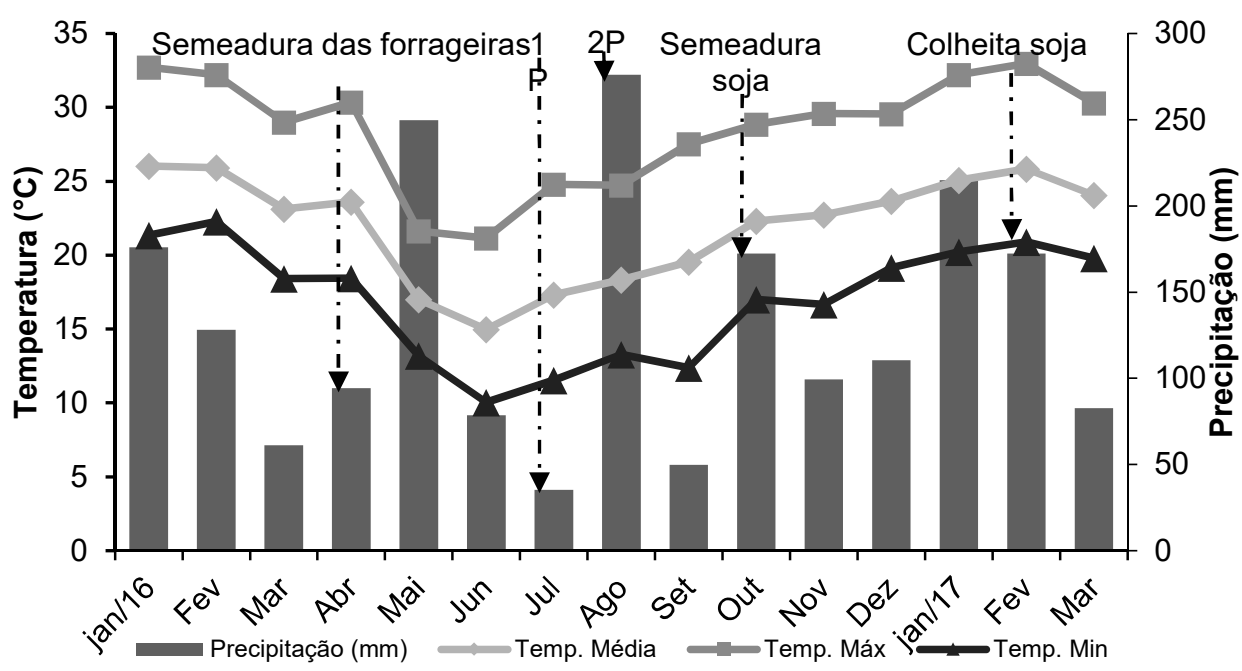

Figura 1. Médias mensais de temperaturas máxima, média e mínima do ar e precipitação pluviométrica acumulada durante o período de condução do experimento. 1P e 2P: pastejos da aveia preta no inverno. Fonte: Estação Climatológica Automática localizada no Núcleo de Estações Experimentais da UNIOESTE, Marechal Cândido Rondon - PR.

Para a implantação do experimento não foi realizado a calagem da área, uma vez que a saturação de bases estava em torno de 50\% (Tabela 1) (PRIMAVESI et al., 2000). Na área experimental foi realizado a dessecação da cultura da soja, que estava presente, com o uso de glyphosate-sal de isopropilamina (i.a.) e clethodim (i.a.) nas doses de $4 \mathrm{~L} \mathrm{ha}{ }^{-1}$ e $0,5 \mathrm{~L} \mathrm{ha}^{-1}$ do produto comercial, respectivamente, com volume de calda de $250 \mathrm{~L} \mathrm{ha}^{-1}$, cerca de 30 dias antes da implantação do experimento.

A semeadura das forrageiras invernais ocorreu no dia 16 de abril de 2016 utilizando a semeadora adubadora de fluxo contínuo, com taxas de semeadura de $60 \mathrm{~kg} \mathrm{ha}^{-1}, 140 \mathrm{~kg} \mathrm{ha}^{-1}$ e $40+120 \mathrm{~kg} \mathrm{ha}^{-1}$ para as aveias, o triticale e o consórcio, respectivamente, diretamente sobre a palhada remanescente da cultura soja e com espaçamento de $0,17 \mathrm{~m}$ entrelinhas. A adubação de semeadura empregada foi de $250 \mathrm{~kg} \mathrm{ha}^{-1} \mathrm{do}$ formulado 10-15-15 (N, $\mathrm{P}_{2} \mathrm{O}_{5}$ e $\mathrm{K}_{2} \mathrm{O}$ ) (PRIMAVESI et al., 2000) e adubação de cobertura de $120 \mathrm{~kg} \mathrm{ha}^{-1}$ de $\mathrm{N}$ na forma de ureia.

Durante o desenvolvimento das forrageiras a adubação de cobertura foi parcelada em duas vezes (60 $\left.\mathrm{kg} \mathrm{ha}^{-1}\right)$ para as parcelas manejadas sem pastejo e um pastejo, e em três vezes $\left(40 \mathrm{~kg} \mathrm{ha}^{-1}\right)$ nas parcelas pastejadas duas vezes. A primeira aplicação foi realizada em 16 e maio de 2016, 30 dias após o plantio; a segunda em 7 de julho de 2016, após o primeiro ciclo de pastejo; e a terceira, em 5 de agosto de 2016, após o segundo pastejo nas parcelas manejadas com dois pastejos. Em relação aos tratos fitossanitários, durante o ciclo das forrageiras não houve necessidade de aplicação de herbicidas, inseticidas e/ou fungicidas.

Os pastejos foram iniciados aproximadamente 90 dias após a emergência das plantas, quando estas estavam entre 0,25 - 0,35 $\mathrm{m}$ de altura e cessados quando a altura de plantas residual estava entre $0,15-0,20$ $\mathrm{m}$, para que não houvesse danos ao meristema apical e para a formação de palhada suficiente para o plantio direto da soja em sucessão. Foram utilizados animais da raça holandesa em fase de lactação com peso médio de $600 \mathrm{~kg}$. Para as parcelas sem a presença de pastejo, essas permaneceram inalteradas até suas adequadas épocas de dessecação.

Para a implantação da cultura de verão a área foi previamente dessecada, 36 dias após o segundo pastejo, utilizando-se glyphosate-sal de isopropilamina + clethodim na dose de $3,0 \mathrm{~L} \mathrm{ha}^{-1}$ e $0,40 \mathrm{~L} \mathrm{ha}^{-1}$ de produto comercial contendo $480 \mathrm{~g} \mathrm{~L}^{-1}$ e $240 \mathrm{~g} \mathrm{~L}^{-1}$ de ingrediente ativo, respectivamente. A semeadura da soja foi realizada em sistema de semeadura direta na palha, no dia 21 de outubro de 2016 utilizando-se a cultivar de NIDERA 5909 RR, com 0,50 m de espaçamento entre linhas, a $4 \mathrm{~cm}$ de profundidade e 14 sementes por metro linear. 
Para a adubação de base foi utilizado $310 \mathrm{~kg} \mathrm{ha}^{-1}$ do formulado comercial 02-20-18 $\left(\mathrm{N}, \mathrm{P}_{2} \mathrm{O}_{5}\right.$ e $\left.\mathrm{K}_{2} \mathrm{O}\right)$ (EMBRAPA, 2003) e durante o desenvolvimento da cultura foram realizadas aplicações de fungicidas Piraclostrobina + fluxapiroxade na dose de $300 \mathrm{~mL} \mathrm{ha}^{-1}$, de produto comercial; e inseticidas: neonicotinóide + piretróide e benzoiluréia, nas doses de $250 \mathrm{~mL} \mathrm{ha}^{-1}$ e $300 \mathrm{~mL} \mathrm{ha}^{-1}$, de produto comercial, respectivamente, com volume de calda de $250 \mathrm{~L} \mathrm{ha}^{-1}$. A colheita da soja foi realizada de forma manual, no dia 27 de fevereiro de 2017 , em pleno estagio de maturação dos grãos (R8).

As coletas das amostras de solo foram realizadas em meados de abril de 2017, após a colheita da soja e cerca de um ano após semeadura das forrageiras. Para a determinação da matéria orgânica, carbono orgânico total e nitrogênio total do solo foram realizadas as coletas de amostras compostas, formada por quatro amostras simples retiradas na área útil da parcela em cada camada, com o auxílio de trado holandês, nas camadas 0-0,05 m, 0,05-0,10 m e 0,10-0,20 m. Após a coleta, as amostras foram acondicionadas em sacos plásticos, identificadas e transportadas ao Laboratório de Física do solo da UNIOESTE onde foram secas ao ar e passadas em peneira de $2 \mathrm{~mm}$ para obtenção da terra fina seca ao ar.

O carbono orgânico total (COT) foi determinado pelo método de oxidação via úmida, com aquecimento externo, segundo método proposto por Yeomans e Bremner (MENDONÇA \& MATOS, 2017). A matéria orgânica do solo (MOS) foi determinada pelo método da mufla (SILVA \& QUEIROZ, 2006), onde a massa de solo foi previamente seca a $100^{\circ} \mathrm{C}$ e em seguida queimada em mufla a $550^{\circ} \mathrm{C}$, atribuindo a perda de massa verificada na secagem a eliminação da matéria orgânica.

O nitrogênio total do solo (NT) foi determinado por destilação de método de Kjeldahl após digestão sulfúrica, conforme descrito por Mendonça e Matos (2017), e a relação carbono e nitrogênio (C/N) estimada a partir dos valores obtidos dos teores de carbono orgânico total e nitrogênio total das amostras.

Foram coletadas três amostras indeformadas por parcela nas camadas de 0-0,05 m, 0,05-0,10 m e 0,10-0,20 m com auxílio de anéis de aço de volume conhecido $\left(100 \mathrm{~cm}^{3}\right)$. Em laboratório foram determinados a densidade aparente do solo (Ds), porosidade total (Pt), macroporosidade (Mac), microporosidade (Mic), pelo método do anel volumétrico de volume em mesa de tensão, conforme descrito por Teixeira et al. (2017).

Para o presente estudo foi considerado três fontes de variação, a saber espécies de forrageiras invernais (forrageiras), manejos de pastejo (manejos) e camadas amostradas (camadas), e o seguinte modelo estatístico foi utilizado:

$$
Y_{i j k z}=\mu+A_{i}+B_{j}+\text { erro } a_{i j}+C_{k}+\text { erro } b_{k j}+D_{z}+A_{i} C_{k}+A_{i} D_{z}+C_{k} D_{z}+A_{i} C_{k} D_{z}+\text { erro } C_{i j k z},
$$

Onde: $Y_{\mathrm{ijkz}}$ é o valor observado a partir do somatório da média $(\mu)$, efeitos das fontes de variação e erros experimentais; A representa as espécies de forrageiras invernais ( $i=1,2,3,4,5)$; B representa os blocos $(j=$ $1,2,3)$; C representa os manejos de pastejo $(k=1,2,3)$; e $D$ representa as camadas amostradas $(z=1,2,3)$.

Os dados obtidos foram submetidos à análise de variância pelo teste $F(p \leq 0,05)$ e quando significativas as médias foram comparadas através do teste de Tukey $(p \leq 0,05)$, utilizando o programa estatístico SAS University Edition (SAS INSTITUTE, 2013).

\section{Resultados e Discussão}

Os atributos químicos NT e MOS foram influenciados pela interação das forrageiras invernais versus manejo de pastejo e para o efeito isolado entre as camadas amostradas, enquanto na relação C/ $\mathrm{N}$ e COT foram observados efeito isolado entre camadas amostradas. Em relação aos atributos físicos foram encontradas interações (forrageiras invernais versus manejos versus camadas) para Mic e Pt, (forrageiras invernais versus camada) para Mac e efeito simples da camada para Ds.

Foram observadas diferenças nos teores do NT e MOS pelo teste de Tukey $(p \leq 0,05)$ ao analisar a interação das forrageiras invernais e manejos, em que a realização de um pastejo sobre a triticale proporcionou melhores resultados quando comparado, a mesma condição de pastejo, a aveia IAPAR 61 (Tabela 2). Essa diferença pode estar a relacionada a decomposição e permanência do material vegetal deixado pelas plantas e, posterior liberação de nitrogênio, na qual a aveia por presentar menor relação C/N que a triticale apresentaria menor permanência de seus resíduos e dos efeitos seus efeitos no solo.

Agrarian, Dourados, v. 13, n. 50, p. 467- 477, 2020. 
Tabela 2. Teores de nitrogênio total (NT), carbono orgânico total (COT), matéria orgânica do solo (MOS) de um LATOSSOLO VERMELHO Eutroférrico cultivado sob manejos plantas forrageiras após a colheita da soja

\begin{tabular}{|c|c|c|c|c|c|c|c|c|c|}
\hline \multirow{3}{*}{ Forrageiras } & \multicolumn{3}{|c|}{ NT $\mathbf{g ~ k g}^{-1}$} & \multicolumn{3}{|c|}{ COT kg $^{-1}$} & \multicolumn{3}{|c|}{ MOS \% } \\
\hline & \multicolumn{6}{|c|}{ - } & \multicolumn{3}{|c|}{$\%$} \\
\hline & SP & $1 \mathrm{P}$ & $2 \mathrm{P}$ & SP & $1 \mathrm{P}$ & $2 \mathrm{P}$ & SP & $1 \mathrm{P}$ & $2 \mathrm{P}$ \\
\hline BRS139 & $1,27 \mathrm{aA}$ & $1,27 \mathrm{aAB}$ & $1,20 \mathrm{aA}$ & $18,1 \mathrm{aA}$ & 17,6 aA & $16,6 \mathrm{aA}$ & $12,0 \mathrm{aA}$ & $11,78 \mathrm{aAB}$ & $12,28 \mathrm{aA}$ \\
\hline Esm & $1,30 \mathrm{aA}$ & $1,22 \mathrm{aAB}$ & $1,17 \mathrm{aA}$ & 18,6 aA & 18,0 aA & $17,0 \mathrm{aA}$ & $12,4 \mathrm{aA}$ & $12,02 \mathrm{aAB}$ & $11,79 \mathrm{aA}$ \\
\hline IPR 61 & $1,30 \mathrm{aA}$ & $1,14 \mathrm{aB}$ & $1,24 \mathrm{aA}$ & $18,7 \mathrm{aA}$ & 17,0 aA & $18,2 \mathrm{aA}$ & $11,9 \mathrm{aA}$ & $11,53 \mathrm{aB}$ & $12,27 \mathrm{aA}$ \\
\hline Triti & $1,26 \mathrm{aA}$ & $1,30 \mathrm{aA}$ & $1,25 \mathrm{aA}$ & $17,7 \mathrm{aA}$ & $18,5 \mathrm{aA}$ & $18,2 \mathrm{aA}$ & $11,7 \mathrm{aA}$ & $12,48 \mathrm{aA}$ & $12,20 \mathrm{aA}$ \\
\hline Cons & $1,25 \mathrm{aA}$ & $1,15 \mathrm{aAB}$ & $1,22 \mathrm{aA}$ & $18,0 \mathrm{aA}$ & 17,3 aA & $18,0 \mathrm{aA}$ & $12,2 \mathrm{aA}$ & $12,08 \mathrm{aAB}$ & $11,97 \mathrm{aA}$ \\
\hline Média geral & 1,28 & 1,22 & 1,22 & 18,2 & 17,7 & 17,6 & 12,0 & 12,0 & 12,1 \\
\hline
\end{tabular}

BRS139: aveia preta BRS 139, Esm: aveia branca IPR Esmeralda; IPR 61: aveia preta IPR 61; Triti: Triticale 111 TPolo; Cons: Consórcio entre aveia branca IPR Esmeralda e Triticale 111 TPolo. SP: sem pastejo; 1P: um pastejo; 2P: dois pastejos. Médias seguidas de mesma letra minúscula na linha e maiúscula na coluna não diferem estatisticamente pelo teste Tukey (5\%).

Para o COT embora tenha-se observado diferenças na interação das forrageiras invernais e manejos não foram encontradas diferenças entre as médias pelo teste de Tukey, isso se deve ao maior rigor que este apresenta ao comparar os tratamentos. A não diferença entre os tratamentos pode estar associada a adubação utilizada que favorece direta ou indiretamente a atividade microbiana decompositora resultando em teores semelhantes entre as áreas (Pinheiro et al., 2013) e ao curto período de condução e avaliação do experimento (MORAES REGO et al., 2020).

Foram observados decréscimo do NT, COT, MOS e C/N conforme aumento da profundidade, tal comportamento é devido a maior deposição de resíduos sobre a superfície do solo, a concentração das raízes das plantas mais próximo da superfície e a presença de dejetos animais que favorecem o aumento da matéria orgânica (Tabela 3). Resultados semelhantes foram encontrados por Santos et al. (2009) ao estudarem o efeito do sistema integrado sobre a fertilidade do solo e Rosset et al. (2016) ao estudarem as frações químicas e oxidáveis da matéria orgânica de diferentes sistemas de manejo. Em geral, os valores encontrados para a relação C/N estão dentro do limite aceitável para solos agricultáveis, que devem variar entre 9 e 14 (CARVALHO et al., 2016).

Tabela 3. Teores de nitrogênio total (NT), carbono orgânico total (COT), matéria orgânica do solo (MOS) e relação carbono por nitrogênio $(\mathrm{C} / \mathrm{N})$ nas camadas de um LATOSSOLO VERMELHO Eutroférrico cultivado em sistema de integração lavoura-pecuária.

\begin{tabular}{lcccc}
\hline Camadas $(\mathbf{m})$ & $\mathbf{N T}\left(\mathbf{g ~ k g}^{-1}\right)$ & COT $\left.\mathbf{( \mathbf { ~ k g } ^ { - 1 }}\right)$ & MOS $\mathbf{( \% )}$ & $\mathbf{C} / \mathbf{N}$ \\
\hline $0-0,05$ & $1,35 \mathrm{a}$ & $19,92 \mathrm{a}$ & $12,32 \mathrm{a}$ & $14,75 \mathrm{a}$ \\
$0,05-0,10$ & $1,24 \mathrm{~b}$ & $17,71 \mathrm{~b}$ & $12,03 \mathrm{~b}$ & $14,28 \mathrm{ab}$ \\
$0,10-0,20$ & $1,12 \mathrm{c}$ & $15,93 \mathrm{c}$ & $11,80 \mathrm{c}$ & $14,22 \mathrm{~b}$ \\
\hline Média geral & 1,24 & 17,8 & 12,05 & 14,4
\end{tabular}

Médias seguidas pela mesma letra na coluna diferem estatisticamente pelo teste de Tukey $(5 \%)$.

A realização de dois pastejos sobre o triticale, na camada 0,05-0,10 m, apresentou os menores valores de Mic quando comparado as demais forrageiras, manejos e camadas. Para Pt foram observadas diferenças entre as camadas $0,05-0,10$ e $0,10-0,20 \mathrm{~m}$, na qual a realização de 1 pastejo sobre aveia esmeralda e triticale, 2 pastejos sobre triticale e sem pastejo para aveia esmeralda e lapar 61 foram observados os menores valores (Tabela 4). 
Tabela 4. Microporosidade e Porosidade Total nas camadas de um LATOSSOLO VERMELHO Eutroférrico cultivado com soja em sucessão a plantas forrageiras.

\begin{tabular}{|c|c|c|c|c|c|c|}
\hline \multirow{2}{*}{ Forrageiras } & \multicolumn{3}{|c|}{ Microporosidade $\left(\mathrm{m}^{3} \mathrm{~m}^{-3}\right)$} & \multicolumn{3}{|c|}{ Porosidade Total $\left(\mathrm{m}^{3} \mathrm{~m}^{-3}\right)$} \\
\hline & SP & 1P & $2 P$ & SP & 1P & $2 P$ \\
\hline & \multicolumn{6}{|c|}{ Camada 0-0,05 m } \\
\hline BRS139 & $0,44 \mathrm{aA \alpha}$ & 0,46 aAa & $0,46 \mathrm{aA \alpha}$ & $0,58 \mathrm{aAa}$ & $0,56 \mathrm{aA \alpha}$ & $0,59 \mathrm{aAa}$ \\
\hline Esm & $0,46 \mathrm{aAa}$ & $0,49 \mathrm{aA \alpha}$ & $0,48 \mathrm{aAa}$ & $0,55 \mathrm{aA \alpha}$ & $0,60 \mathrm{aA \alpha}$ & $0,52 a A \alpha$ \\
\hline IPR 61 & 0,45 aAa & 0,46 aAa & $0,44 \mathrm{aA} \alpha$ & $0,62 \mathrm{aAa}$ & 0,58 aAa & $0,55 \mathrm{aA \alpha}$ \\
\hline Triti & 0,47 aAa & $0,43 a A \alpha$ & 0,48 aAa & $0,58 \mathrm{aA \alpha}$ & $0,64 \mathrm{aAa}$ & $0,62 \mathrm{aA} \alpha$ \\
\hline \multirow[t]{2}{*}{ Cons } & $0,42 \mathrm{aAa}$ & $0,49 \mathrm{aA \alpha}$ & $0,46 \mathrm{aAa}$ & $0,57 \mathrm{aAa}$ & $0,57 \mathrm{aA \alpha}$ & $0,59 \mathrm{aAa}$ \\
\hline & \multicolumn{6}{|c|}{ Camada $0,05-0,10 \mathrm{~m}$} \\
\hline BRS139 & $0,42 \mathrm{aA \alpha}$ & $0,43 a A \alpha$ & $0,44 \mathrm{aA \alpha}$ & $0,51 \mathrm{aA \alpha}$ & $0,53 \mathrm{aA \alpha}$ & $0,54 \mathrm{aAa}$ \\
\hline Esm & $0,43 \mathrm{aAa}$ & $0,43 a A \alpha$ & $0,45 \mathrm{aAa}$ & $0,51 \mathrm{aA} \beta$ & $0,51 \mathrm{aA} \beta$ & $0,49 a A a$ \\
\hline IPR 61 & $0,42 \mathrm{aAa}$ & $0,42 \mathrm{aA \alpha}$ & $0,43 \mathrm{aA \alpha}$ & $0,51 \mathrm{aA} \beta$ & $0,51 \mathrm{aA \alpha}$ & $0,53 \mathrm{aA \alpha}$ \\
\hline Triti & $0,45 \mathrm{aA \alpha}$ & $0,43 \mathrm{aA \alpha}$ & $0,41 \mathrm{aA} \beta$ & $0,55 \mathrm{aA \alpha}$ & $0,53 a A \beta$ & $0,51 \mathrm{aA} \beta$ \\
\hline \multirow[t]{2}{*}{ Cons } & $0,41 \mathrm{aAa}$ & $0,42 \mathrm{aAa}$ & $0,43 \mathrm{aAa}$ & $0,51 \mathrm{aAa}$ & $0,52 \mathrm{aAa}$ & $0,52 \mathrm{aAa}$ \\
\hline & \multicolumn{6}{|c|}{ Camada $0,10-0,20 \mathrm{~m}$} \\
\hline BRS139 & $0,44 \mathrm{aA \alpha}$ & $0,44 \mathrm{aA \alpha}$ & 0,43 aAa & $0,53 a A \alpha$ & $0,53 \mathrm{aA \alpha}$ & $0,53 a A \alpha$ \\
\hline Esm & $0,41 \mathrm{aA \alpha}$ & $0,42 \mathrm{aA \alpha}$ & 0,49 aAa & 0,51 aA $\beta$ & 0,52 aA $\beta$ & 0,53 aAa \\
\hline IPR 61 & $0,42 a A \alpha$ & $0,41 \mathrm{aA \alpha}$ & $0,43 a A \alpha$ & $0,52 \mathrm{aA} \beta$ & $0,52 \mathrm{aAa}$ & $0,55 a A \alpha$ \\
\hline Triti & $0,42 \mathrm{aAa}$ & $0,43 a A \alpha$ & $0,42 a A \alpha \beta$ & $0,50 a A \alpha$ & $0,52 \mathrm{aA} \beta$ & $0,51 \mathrm{aA} \beta$ \\
\hline Cons & $0,42 \mathrm{aA} \alpha$ & $0,43 a A \alpha$ & 0,45 aAa & $0,51 \mathrm{aA \alpha}$ & $0,54 \mathrm{aA} \alpha$ & $0,52 \mathrm{aAa}$ \\
\hline
\end{tabular}

BRS139: aveia preta BRS 139, Esm: aveia branca IPR Esmeralda; IPR 61: aveia preta IPR 61; Triti: Triticale 111 TPolo; Cons: Consórcio entre aveia branca IPR Esmeralda e Triticale 111 TPolo. SP: sem pastejo; 1P: um pastejo; 2P: dois pastejos. Médias seguidas da mesma letra minúscula na linha, maiúscula na coluna e letras gregas entre as camadas não diferem estatisticamente pelo teste de Tukey a $5 \%$ de probabilidade.

De modo geral, o pisoteio animal interferiu de forma pouco expressiva sobre os atributos físicos estudadas, corroborando com os resultados observados por Kunz et al. (2013) e Bortolini et al. (2016), sendo possível observar que a realização de maiores frequências de manejo não acarretou mudanças sobre as camadas avaliadas, em especial sobre o emprego do consórcio de forrageiras e aveia BRS139. Kunz et al. (2013) ao analisarem a compactação do solo na integração soja-pecuária também constataram o aumento da microporosidade na camada superficial em áreas de plantio direto com pastejo de aveia corroborando com os resultados encontrados.

Ao considerar os valores de microporosidade e porosidade total, observa-se que estes estiveram acima de $0,40 \mathrm{~m}^{3} \mathrm{~m}^{-3}$ e $0,50 \mathrm{~m}^{3} \mathrm{~m}^{-3}$, respectivamente, enquanto a macroporosidade em alguns casos inferior a 0,10 $\mathrm{m}^{3} \mathrm{~m}^{-3}$. Contudo, nota-se que esse comportamento foi independente do manejo adotado.

$\mathrm{Na}$ Mac foram observadas diferenças nas forrageiras na camada 0-0,5 m, onde as aveias BRS139 e lapar 61 e a triticale apresentarem os maiores valores (Tabela 5). Foi observado no consorcio diminuição nos valores da Mac na camada superficial e nas demais apresentaram comportamento semelhante. Os valores de Mac encontrados estão abaixo do ideal $\left(<0,10 \mathrm{~m}^{3} \mathrm{~m}^{-3}\right)$ em todas as camadas do solo, independente da forrageira ou manejo adotado, podendo ser reflexo das pressões exercidas pelo tráfico de máquina dentro da área e manejos anteriores (SILVA et al., 2012; PIANO et al., 2015). 
Tabela 5. Macroporosidade do solo nas camadas de um LATOSSOLO VERMELHO Eutroférrico cultivado com plantas forrageiras e soja em sucessão.

\begin{tabular}{|c|c|c|c|}
\hline \multirow{3}{*}{ Forrageiras } & \multicolumn{3}{|c|}{ Macroporosidade $\left(\mathrm{m}^{3} \mathrm{~m}^{-3}\right)$} \\
\hline & \multicolumn{3}{|c|}{ Camada (m) } \\
\hline & $0-0,05$ & $0,05-0,10$ & $0,10-0,20$ \\
\hline BRS139 & 0,1269 aA & $0,0967 \mathrm{abA}$ & $0,0944 \mathrm{bA}$ \\
\hline Esm & $0,1205 \mathrm{aAB}$ & 0,0868 aA & $0,1013 \mathrm{aA}$ \\
\hline IPR 61 & 0,1240 aA & 0,0933 aA & 0,1089 aA \\
\hline Triti & 0,1406 aA & $0,0967 \mathrm{bA}$ & $0,0867 \mathrm{bA}$ \\
\hline Cons & $0,0873 \mathrm{aB}$ & 0,0911 aA & $0,0971 \mathrm{aA}$ \\
\hline Média geral & 0,1198 & 0,0930 & 0,0976 \\
\hline
\end{tabular}

BRS139: aveia preta BRS 139, Esm: aveia branca IPR Esmeralda; IPR 61: aveia preta IPR 61; Triti: Triticale 111 TPolo; Cons: Consórcio entre aveia branca IPR Esmeralda e Triticale 111 TPolo. Médias seguidas da mesma letra minúscula na linha e maiúscula na coluna não diferem estatisticamente entre si pelo teste de Tukey (5\%).

Piano et al. (2015) avaliando atributos físicos do solo submetido aos cultivos com aveia, trigo e triticale manejados sem pastejo, um pastejo ou dois pastejos, observaram alterações na Mac na camada de 0-0,10 m, e Mic e Pt ambos nas camadas de 0,10-0,20 m após o cultivo da soja atrelando seus resultados as pressões exercidas pela entrada dos animais na área, embora as diferenças tenham sido pequenas. Ainda segundo esses autores, os valores médios de Mac, Mic e Pt encontrados foram de $0,07 \mathrm{~m}^{3} \mathrm{~m}^{-3}, 0,48 \mathrm{~m}^{3} \mathrm{~m}^{-3}$ e $0,55 \mathrm{~m}^{3} \mathrm{~m}$ ${ }^{3}$, respectivamente, na camada de $0,0-0,10 \mathrm{~m} \mathrm{e} 0,06 \mathrm{~m}^{3} \mathrm{~m}^{-3}, 0,46 \mathrm{~m}^{3} \mathrm{~m}^{-3}, 0,52 \mathrm{~m}^{3} \mathrm{~m}^{-3}$, respectivamente, na camada de 0,10-0,20 valores próximos aos encontrados no presente estudo.

A Ds apresentou diferença entre as camadas, não sendo influenciada pelas forrageiras e manejos empregados (Tabela 6), tais resultado indicam que a realização de pastejo sobre área comercial de grão não afetaria a Ds, sendo uma opção de diversificação produtiva durante o período de inverno aos produtores. Piano et al. (2015) também não encontraram efeitos na densidade do solo em função dos manejos (sem pastejo, um pastejo e dois pastejos) e culturas (aveia, trigo e triticale).

Tabela 6. Densidade do solo nas camadas de um LATOSSOLO VERMELHO Eutroférrico conduzido em sistema de integração lavoura-pecuária após cultivo de plantas forrageiras e soja em sucessão.

\section{Camada (m)}

\section{$0-0,05$}

$0,05-0,10$

$0,10-0,20$

Média geral

Médias seguidas de mesma letra minúscula não apresentam diferença estatística pelo teste de Tukey (5\%).

\section{Densidade $\left(\mathrm{Mg} \mathrm{m}^{-3}\right)$}

$1,18 \mathrm{~b}$

$1,30 \mathrm{a}$

$1,29 \mathrm{ab}$

Nota-se que a camada superficial expressa densidade de $1,18 \mathrm{Mg} \mathrm{m}^{-3}$, enquanto as camadas mais profundas atingiram valores próximos a $1,33 \mathrm{Mg} \mathrm{m}^{-3}$, considerado crítico para este solo por Klein e Câmara (2007). A densidade do solo quando elevada restringe o crescimento radicular das plantas, principalmente em períodos secos, onde o acesso à água e aos nutrientes das camadas mais profundas são limitados (COLLARES et al., 2008).

O fato da Ds na camada superficial ser inferior às camadas inferiores, pode ser justificado pelo elevado teor de matéria orgânica e presença dos resíduos vegetais sobre o solo, pois de acordo com Braida et al. (2006) a matéria orgânica é menos densa que a fração mineral e a presença de palha sobre o solo absorve parte da energia de compactação produzida pelo trânsito de máquinas e animais. Contudo, os autores ressaltam que a capacidade de absorção do impacto depende da quantidade de palha, da energia aplicada e das condições do solo.

Resultados distintos foram encontrados por Ortigara et al. (2014) ao avaliarem os atributos físicos e mecânicos de um Latossolo manejado sob pastejo rotacionado, preparo convencional e mata, observaram que

Agrarian, Dourados, v. 13, n. 50, p. 467- 477, 2020. 
tanto a densidade do solo como a resistência à penetração foram influenciadas negativamente pelo pisoteio animal, resultando em níveis limitantes ao crescimento radicular das plantas.

\section{Conclusões}

As forrageiras invernais quando submetidas a maiores pressões de pastejo não modificam os atributos químicos do solo.

O Triticale 111 Tpolo quando submetidas a menor pressão de pastejo proporcionou melhores resultados sobres os atributos químicos NT e MOS que a aveia IAPAR 61.

O emprego da aveia BRS139 e no consorcio de Triticale mais aveia Esmeralda e a maior pressão de pastejo não modificaram aos atributos físicos do solo.

\section{Agradecimentos}

Este estudo foi financiado em parte pela Coordenação de Aperfeiçoamento de Pessoal de Nível Superior - Brasil (CAPES) - Código Financeiro 001. Queremos agradecer à Coordenação de Aperfeiçoamento de Pessoal de Nível Superior (CAPES) pela bolsa de estudos e recursos para realização da pesquisa e ao Conselho Nacional de Desenvolvimento Científico e Tecnológico (CNPq) pela bolsa de produtividade concedida ao sexto autor.

\section{Referências}

ALVARES, C.A.; STAPE, J.L.; SENTELHAS, P.C.; DE MORAES GONÇALVES, J.L.; SPAROVEK, G. Köppen's climate classification map for Brazil. Meteorologische Zeitschrift, v.22, n.6, p.711-728, 2014.

BALBINO, L.C.; KICHEL, A.N.; BUNGENSTAB, D.J.; ALMEIDA, R.G. Sistemas de integração: conceitos, considerações, contribuições e desafios. In: BUNGENSTAB, D.J.; ALMEIDA, R.G. de; LAURA, V.A.; BALBINO, L.C.; FERREIRA, A.D. (Ed.). ILPF: inovação com integração de lavoura, pecuária e floresta. Brasília, Embrapa, 2019. p.31-48.

BORTOLINI, D.; ALBUQUERQUE, J.A.; RECH, C.; MAFRA, A.L.; RIBEIRO FILHO, H.M.N.; PÉRTILE, P. Propriedades físicas do solo em sistema de integração lavoura-pecuária em Cambissolo Húmico. Revista de Ciências Agroveterinárias, v. 15, n. 1, p. 60-67, 2016.

BRAIDA, J.A.; REICHERT, J.M.; VEIGA, M.; REINERT, D.J. Resíduos Vegetais na superfície e carbono orgânico do solo e suas relações com a densidade máxima obtida no ensaio proctor. Revista Brasileira de Ciência do Solo, v. 30, n. 4, p. 605-614, 2006.

CARVALHO, J.S.; KUNDE, R.J.; STÖCKER, C.M.; LIMA, A.C.R.; SILVA, J.L.S. Evolution of physical, chemical, and biological attributes of hydromorphic soil under crop-livestock integration systems in the Pampa biome. Pesquisa Agropecuária Brasileira, v.51, n.9, p.1131-1139, 2016.

CARVALHO, P.C.F.; MORAES, A.; PONTES, L.S.; ANGHINONI, I.; SULC, M.R.; BATELLO, C. Definitions and terminologies for Integrated Crop-Livestock System. Revista Ciência Agronômica, v. 45, n.5, p. 1040-1046, 2014.

COLLARES, G.L.; REINERT, D.J.; REICHERT, J.M; KAISER, D.R. Compactação de um Latossolo induzida pelo tráfego de máquinas e sua relação com o crescimento e produtividade de feijão e trigo. Revista Brasileira de Ciência do Solo, v. 32, n. 3, p.933-942, 2008.

COSTA, N.R.; ANDREOTTI, M.; MASCARENHAS, K.S.L.; YOKOBATAKE, L.K. FERREIRA, J.P.; PARIZ, M.C.; dos SANTOS, C.B.B.; LONGHINI, V.Z. Atributos do solo e Acúmulo de Carbono na Integração LavouraPecuária em Sistema Plantio Direto. Revista Brasileira de Ciência do Solo, v. 39, n. 3, p. 852-863, 2015.

EMBRAPA. Tecnologias de produção de soja Paraná 2004. Londrina: Embrapa Soja, Sistema de Produção, 2003. 218p. 
GLÉRIA, A.A.; SILVA, R.M.; SANTOS, A.P.P.; SANTOS, K.J.G.; PAIM, T.P. Produção de bovinos de corte em sistemas de integração lavoura pecuária. Archivos de Zootecnia, v. 66, n. 253, p. 141-150, 2017.

KLEIN, V.A.; CAMARA, R.K. Rendimento da soja e intervalo hídrico ótimo em Latossolo Vermelho sob plantio direto escarificado. Revista Brasileira de Ciência do Solo, v. 31, p. 221-227, 2007.

KUNRATH, T.R.; CARVALHO, P.C.F. DE; CADENAZZI, M.; BREDEMEIER, C.; ANGHINONI, I. Grazing management in an integrated crop-livestock system: soybean development and grain yield. Revista Ciências Agrárias, v.46, n. 3, p. 645-653, 2015.

KUNZ, M.; GONÇALVES, A. D. M. A.; REICHERT, J. M.; GUIMARÃES, R. M. L.; REINERT, D. J.; RODRIGUES, M. F. Compactação do solo na integração soja-pecuária de leite em Latossolo Argiloso com semeadura direta e escarificação. Revista Brasileira de Ciência do Solo, v. 37, n. 6, p. 1699-1708, 2013.

MENDONÇA, E.S.; MATOS, E.S. Matéria orgânica do solo: métodos de análises. 2. ed. rev. atual. Viçosa: UFV, Gefert, 2017. 221 p.

MORAES REGO, C.A.R.; OLIVEIRA, P.S.R.; PIANO, J.T.; ROSSET, J. S.; EGEWARTH, J.F.; MATTEI, E.; SAMPAIO, M.C.; HERRERA, J.L. Organic Matter Fractions and Carbon Management Index in Oxisol Under Integrated Agricultural Production Systems. Journal of Agricultural Studies, v. 8, n. 3, p. 237-256, 2020.

MOREIRA, W. H.; TORMENA, C. A.; BETIOLI JUNIOR, E.; PEATEAN, L. P.; ALVES, S. J. Influência da altura de pastejo de azevém e aveia em atributos físicos de um Latossolo Vermelho Distroférrico, após sete anos sob integração lavoura-pecuária. Revista Brasileira de Ciência do Solo, v. 38, n. 4, p. 1315-1326, 2014.

ORTIGARA, C.; KOPPE, E.; da LUZ, F. B.; KAISER, D. R.; da SILVA, V.R. Uso do solo e propriedades físicomecânicas de Latossolo Vermelho. Revista Brasileira de Ciência do Solo, v. 38, n. 2, p. 619-626, 2014.

PAVAN, M.A.; BLOCH, M.D.M.; ZEMPULSK, H.C.; MIYAZAWA, M.; ZOCOLER, D.C. Manual de análises químicas de solo e controle da qualidade. Londrina: IAPAR, 1992. 40p. (Circular Técnica, n.76).

PIANO, J.T.; OLIVEIRA, P.S.R.; COSTA, P.F.; TAFFAREL, L.E.; EGEWARTH, J.F.; SEIDEL, E.P.; CASTAGNARA, D.D.; BORSOIL, A.; EGEWARTH, V.A. Soil physical atributes under different grazing management of winter forage crops in crop-livestock system at Southern Brazil. African Journal of Agricultural Research, v. 10, n. 3, p. 146-153, 2015.

PINHEIRO, R.M.B.; LOSS, A.; PEREIRA, M.G.; PERIN, A. Formas de carbono em Latossolo sob sistemas de plantio direto e integração lavoura-pecuária no cerrado. Semina: Ciências Agrárias, v. 34, n. 6, p. 2637-2654, 2013.

PRIMAVESI, A.C.; RODRIGUES, A. DE A.; GODOY, R. Recomendação técnica para o cultivo de aveia. Boletim de pesquisa. São Carlos: Embrapa Pecuária Sudeste, 2000. 39p. (Boletim de Pesquisa, 06).

REGO, C.A.R.M.; MUNIZ, L.C.; REIS, V.R.R.; CANTANHEDE, I.S.L.; COSTA, B.P.; MARQUES, E.O.; OLIVEIRA, P.S.R. Análise econômica da implantação de diferentes sistemas de integração lavoura-pecuáriafloresta no município de Pindaré-Mirim, Maranhão. Sodebrás, v.13, n. 146, p. 114-118, 2018.

RODRIGUES, L.M.; TEODORO, A.G.; SANTOS, A.J.M.; BACKES, C.; ROCHA, J.H.T.; GIONGO, P.R.; DOS SANTOS, Y.L.A. Integration Crop-Livestock-Forestry: Interaction between Components and System Sustainability. Archivos de Zootecnia, v.68, n.263, p.448-455, 2019.

ROSSET, J.S.; LANA, M.C.; PEREIRA, M.G.; SCHIAVO, J.A.; RAMPIM, L.; SARTO, M.V.M. Chemical and oxidizable fractions of soil organic matter under different management systems in an Oxisol. Pesquisa Agropecuária Brasileira, v.51, n.9, p.1529-1538, 2016.

SANTOS, H.G.; JACOMINE, P.K.T.; ANJOS, L.H.C.; OLIVEIRA, V.A.; LUMBRERAS, J.F.; COELHO, M.R.; ALMEIDA, J.A.; ARAUJO FILHO, J.C.; OLIVEIRA, J.B.; CUNHA, T.J.F. Sistema Brasileiro de Classificação de Solos. 5. ed. rev. e ampl. Brasília: Embrapa, 2018. 356p. 
SANTOS, H.P.; FONTANELI, R.S.; SPERA, S.T.; TOMM, G.O. Efeito de sistemas de produção integração lavoura-pecuária (ILP) sobre a fertilidade do solo em plantio direto. Acta Scientiarum Agronomy, v. 31, n. 4, p. 719-727, 2009.

SILVA, C.A.T.; CEZAR, T.C.M.; NÓBREGA, L.H.P. Porosity of oxisols and management practices for agricultural soil conservation. Revista Varia Scientia Agrárias, v.02, n.02, p.153-164, 2012.

SILVA, D.J.; QUEIROZ, A.C. Análise de alimentos: métodos químicos e biológicos. 3.ed. Viçosa: UFV, 2006. ISBN: 85-7269-105-7.

STATISTICAL ANALYSIS SYSTEM - SAS. User's guide. Cary: SAS Institute, 2002. 525 p.

TEIXEIRA, P.C.; DONAGEMMA, G.K.; FONTANA, A.; TEIXEIRA, W.G. Manual de métodos de análises de solo. 3. ed. rev. e ampl. Brasília: Embrapa, 2017. 573 p. 